\title{
Calistenia: regreso a los métodos clásicos de acondicionamiento físico
}

Calisthenics: returning to classic methods of physical conditioning

\author{
Pablo Subires-Gómez ${ }^{1}$; Sanja Starcevic ${ }^{2}$ \\ ${ }^{1}$ Egresado de la Facultad de Ciencias de la Educación, Universidad de Málaga, Málaga, España \\ ${ }^{2}$ Grand Koliba trainer, Bratislava, Slovakia
}

\section{Detalles del artículo:}

Número de palabras:3.821; Tablas: 0; Figuras: 0; Referencias: 17

Recibido: julio 2021; Aceptado: septiembre 2021; Publicado: diciembre 2021

Conflicto de interés: El autor declara que no existen conflictos de interés.

Correspondencia del autor: Pablo Subires Gómez, pablosubiresgomez@gmail.com

\section{Resumen}

Considerando el contexto actual por el que atraviesa la actividad física en el que se trata de imponer un cierto de tipo de método de entrenamiento, con un objetivo más focalizado hacia lo estético, este artículo presenta tanto una descripción como una reflexión de esta alternativa urbana de ejercicio al aire libre, como es la antiquísima calistenia. Es una opción asequible para todo el mundo y aun teniendo alguna desventaja, presenta gran cantidad de beneficios a nivel físico y fisiológico que son fundamentales para el buen mantenimiento de la salud en un ambiente globalizado en el que enfermedades derivadas del sedentarismo son un hecho constante entre sus habitantes. Este artículo tiene como finalidad fomentar hábitos saludables a través de la práctica de la calistenia, actividad que no requiere cuotas ni materiales demasiado sofisticados como ocurre en las salas de musculación. calistenia.

Palabras clave: peso corporal, aptitud física, entrenamiento, deporte, parques

\section{Abstract}

Considering the current context through which physical activity is mischievous in our society in which it is about imposing a certain type of training method with a more focused objective towards aesthetics due to existing patterns, this article presents both a description and a reflection of a different alternative such as the ancient calisthenics that turns out to be an affordable option for everyone and that, even though it has some disadvantage requires a great diversity of optimal benefits both at a physical and physiological level that are fundamental for good maintenance of health in a globalized environment in which diseases derived from sedentary lifestyle are a constant fact among its inhabitants.

Key Words: body weight, fitness, training, sport, calisthenics parks. 


\section{CONTEXTO ACTUAL DE LA ACTIVIDAD FÍSICA}

Actualmente, nuestra sociedad sigue los patrones de un globalismo desmesurado que además de afectar a nuestra vida familiar, social, laboral o académica también influye directamente sobre nuestra manera de mantener nuestra salud en lo que respecta a la realización de actividad física. Sobre la misma, las redes sociales hacen prevalecer una serie de cánones que inciden sobre la población, especialmente con reseñable calado entre las personas jóvenes. La industria del fitness se publicita constantemente a través de las redes sociales que resultan ser una gran fuente de información que a veces no es tratada adecuadamente por los usuarios que acceden a ella.

Aunque generalmente esta industria trata por un lado de implementar la salud a nivel social, por otro lado, hace imperar ciertos patrones repetitivos en relación con la manera y los objetivos de los entrenamientos haciendo destacar pretensiones puramente estéticas y formas de entrenar específicamente analíticas que nada tienen que ver con nuestra naturaleza motriz y funcional. Así pues, desde hace algún tiempo se viene planteando sistemas generalizados de entrenamiento que radican en rutinas alimenticias no equilibradas, excesiva preocupación por la apariencia física y una ejercitación de índole analítica principalmente a través de máquinas en salas de musculación precisando de objetivos conformados en ciclos de hipertrofia y de definición muscular.

Este actual contexto que presenta el ámbito de la actividad física en la sociedad es motivo constante de preocupación y de debate entre profesionales de la Educación Física que observan como las finalidades educativas en relación con el fomento de ámbitos saludables como un adecuado desarrollo motriz o aspectos de introducción deportiva fracasan notoriamente siendo sustituidas por estas perspectivas que se alejan del significado de los valores del deporte y de la Educación Física. Haciendo referencia a lo anterior, observamos que socialmente el entrenamiento de la condición física y especialmente el entrenamiento de fuerza obedece a una serie de patrones más ligados a la práctica del culturismo derivando a que se dejen a un lado otros ciertos aspectos físicos que son cruciales en las funciones físicas como el equilibrio, la coordinación, agilidad, velocidad o la flexibilidad entre otras muchas otras.

Desde sus comienzos, el ser humano, para garantizar su supervivencia ha necesitado de sus capacidades físicas para desplazarse y conseguir alimentos. Hoy día, esto no es necesario por las comodidades de las que disfrutamos y por el modelo de vida que seguimos, aunque un adecuado mantenimiento de nuestra salud física es crucial para mejorar nuestra calidad de vida y no necesariamente ha de estar sujeto a estos principios mencionados en líneas anteriores. De hecho, hace 2500 años en la Antigua Grecia, el ejercicio y el deporte eran las bases de su educación y se tenía un gran interés por el cuidado de la salud física. Tanto militares como atletas que competían en los Juegos, empleaban el método de entrenamiento de la calistenia, ya que no disponían del material tan sofisticado ni la abundancia material superflua actual, y, aun así, mantenían un estado físico óptimo que reflejaron a golpe de cincel en las esculturas que dejaron para la posteridad.

Tras lo expuesto con anterioridad en referencia al rol que ocupan las redes sociales con respecto a los modos de entrenamiento, se plantea una comparativa constante sobre si es mejor usar cargas externas o el propio peso corporal para el entrenamiento de fuerza siendo esta última 
forma la menor valorada. Por ello, en las líneas siguientes se expone una descripción en lo que concierne a la práctica de dicho método.

\section{LA CALISTENIA}

Antes de tratar el concepto de calistenia, se ha de aludir al concepto de fuerza, pues respecto al mismo, hallamos acepciones diferentes. Según Cuadrado et al. (2006) se entiende como aquella capacidad de un individuo para vencer o soportar una resistencia, aunque Knuttgen y Kraemmer (1987) citados en Cuadrado et al. (2006) la conciben como la capacidad de tensión que capaz de generar cada área muscular contra una resistencia con una velocidad concreta de ejecución específica. Por otro lado, según González-Badillo (1991) citado en Alijas y Díaz (2015), la fuerza se define como la capacidad de vencer, equilibrar o frenar el movimiento de una resistencia externa mediante una tensión muscular siendo esta acepción que incluye la calistenia como método adecuado pues en ella se producen estas tres situaciones biomecánicas.

En lo que respecta a la ejercitación de la fuerza, de forma general, Sánchez (2017) estima que el entrenamiento de fuerza es determinado por una serie de variables como son el volumen (normalmente expresado como número de repeticiones); la intensidad (normalmente expresada como porcentaje de 1 repetición máxima (1RM) o como número máximo de repeticiones por serie (XRM); tipo y orden de los ejercicios; relación entre el tiempo de trabajo y descanso (Fleck \& Kraemer, 1987, citados en Sánchez, 2017; Kraemer \& Ratamess, 2004); número de repeticiones efectuados en relación con el máximo número de repeticiones realizables en la serie, es decir, carácter del esfuerzo (González-Badillo, 1992, citado en Sánchez, 2017); la velocidad de ejecución (González-Badillo \& Gorostiaga, 2002; González-Badillo \& Ribas, 2002; González-Badillo, Marqués \& Sánchez-Medina, 2011) y la disminución de velocidad (González-Badillo, Marqués \& Sánchez-Medina, 2011).

Debido a que el sistema neuromuscular tiende hacia la adaptación concreta al estímulo al que es sometido (Coffey \& Hawley, 2007), las adaptaciones fisiológicas y mecánicas que se producen como respuesta al estímulo que supone el entrenamiento de fuerza dependerán de la forma en la que se vinculen dichas variables (Bird, Tarpenning \& Marino, 2005, citados en González-Badillo, 2011; Spiering et al., 2008, citados en González-Badillo, Marqués, \& Sánchez-Medina, 2011). Polo (2020) refleja que el término calistenia deriva del griego y alude al kallos (belleza) y sthenos (fortaleza), podría traducirse como "la belleza del cuerpo en movimiento", es una forma de ejercicio físico consistente en entrenar con el propio peso corporal. Según Alijas y Díaz (2015), la versión más contemporánea de la calistenia se remonta al año 1785 con el profesor de gimnasia Christian Carl André que realizaba esta práctica en la escuela cuando las condiciones climáticas no eran las apropiadas para desarrollar las clases al aire libre, aunque hoy día, esta manera de entrenamiento se lleva a cabo sobre todo en espacios abiertos.

En 1829, Clías, tras publicar su libro "Kallistenie", establece una diferencia terminológica entre Gimnasia y Calistenia, siendo realmente la calistenia una nueva escuela gimnástica en la que se suprimen los aparatos y se adaptaba el ritmo (Polo, 2020), que era diferente a la concepción actual, enfocándose en ejercicios con autocargas y variación de amplitudes que trataban de propiciar un desarrollo integral del cuerpo, con un entrenamiento de 
ejercicios excéntricos, concéntricos e isométricos de fuerza potencia y fuerza resistencia. Por su parte, Harrison (2010) señala que hay más de 600 músculos, todos los cuales desempeñan su función de forma conjunta. Los músculos funcionan con las palancas creadas a través de los huesos y las articulaciones provocando movimiento. Con el entrenamiento con peso corporal, los movimientos no se emplean para mover equipos, sino que son usados para mover el cuerpo contra las fuerzas de la gravedad y las fuerzas de reacción del suelo. De la misma forma que otros tipos de técnicas tradicionales de entrenamiento, como mancuernas o balones medicinales, el entrenamiento con peso corporal es de carácter funcional, puesto que posibilita a la persona trabajar en un contexto tridimensional para vencer la fuerza de la gravedad.

Según Alijas y Díaz (2015), en el transcurso del tiempo, la calistenia ha sido trascendental tanto a nivel deportivo como a nivel social, siendo usada en todas partes para todo tipo de pretensiones y empleada por organizaciones militares para sus entrenamientos. Entre los ejemplos más destacados sobre el empleo de la calistenia para los entrenamientos muestran al campeón olímpico Kashiwa, el cual usó la calistenia como sistema de mejora de la resistencia y potencia. De manera más reciente, en los JJOO de Invierno de Sochi 2014, otro campeón olímpico llamado Félix Neureuther se entrenó con este tipo de método. De acuerdo con Polo (2020), los tipos de calistenia que se pueden llegar a encontrar son muy diferentes. Sánchez (2017) determina que las maneras con mayor predominancia para efectuar calistenia son el trabajo de series y repeticiones, ejercicios dinámicos o trabajo de tensión haciendo alusión como tensión a las posiciones de contracción isométrica.

En referencia con la distinción de los ejercicios aludidos con anterioridad, cabe decir que, pese a que se realice una distinción entre lo que sería un entrenamiento con series y repeticiones y un trabajo de ejercicios dinámicos, estos dos tipos de ejercicios se asocian con lo que serían los ejercicios de tensión. Teniendo en cuenta la concepción de Tillin, Pain \& Folland (2012), esto se debe al tipo de contracción muscular que se efectúan. Los ejercicios de series y repeticiones y los ejercicios dinámicos se trabajan tanto en contracciones de tipo concéntrico y excéntrico, aunque no de la misma manera y con la diferencia de control corporal, postural, nivel de dificultad entre ambas y riesgo de lesión más alto en los ejercicios dinámicos. Según Polo (2020), los ejercicios de tensión son los que prevalecen por sus contracciones de tipo isométrico sobre todo el cuerpo y su gran dificultad para adquirir un alto nivel. De todos los tipos de ejercicios, con total distinción entre los ejercicios de mayor complejidad se destacan los ejercicios de tensión (también denominados ejercicios de presión).

Así pues, como se ha expuesto anteriormente, la calistenia no tiene por qué precisar de contraindicaciones que repercutan negativamente a la salud, puesto que su práctica es totalmente completa y equilibrada, pero como cualquier modalidad deportiva puede llegar a realizarse de manera errónea (Polo, 2020). En referencia a las ventajas, la calistenia se puede llevar a cabo en cualquier lugar, pues al emplear nuestro propio cuerpo, no necesitamos de instalaciones ni tampoco de una inversión económica para material ni cuotas. Por su parte, Harrison (2010) comenta que a pesar de que evidentemente los métodos de entrenamiento con pesas o máquinas pueden hacer que un sujeto sea más fuerte, ejercicios como por ejemplo el curl de bíceps, es un tipo de ejercicio de cadena abierta, ya que emplea sólo una articulación de manera que la resistencia se mueve hacia afuera o hacia el cuerpo utilizando extremidades que se desplazan libremente al tiempo que las fuerzas transmitidas a la articulación afectada incrementan. 
En relación con las máquinas de fuerza, debido a los cambios individuales tanto de tamaño como de fuerza, es complicado fabricar una máquina que se pueda amoldar a las características antropométricas de cada persona (Harrison, 2010). El entrenamiento con el peso corporal, por otra parte, es concreto para la longitud de las extremidades, inserciones músculotendinosas y el peso corporal de cada persona. Además, una gran parte de los ejercicios de peso corporal son ejercicios de cadena cerrada, ya que múltiples articulaciones se involucran a medida que la resistencia se aleja o se acerca a una parte del cuerpo fijada y estos movimientos tienen un carácter más funcional.

El debate acerca de si es mejor emplear el peso corporal o las pesas en una sesión de entrenamiento, es un debate muy frecuente, y por ello, Harrison (2010) concluye determinando que el entrenamiento con el peso corporal no produce ganancias significativas en la fuerza absoluta que se entiende como la capacidad de un individuo para mover una carga absoluta dada. Sin embargo, por otro lado, el entrenamiento con el peso corporal desarrolla la fuerza relativa, definida como la capacidad de una persona para mover una carga como un cierto porcentaje de su peso corporal. Un sujeto que emplea ejercicios de peso corporal utiliza su propio peso como medio de resistencia. Este tipo de entrenamiento de "fuerza relativa", aunque no viene a sustituir a los levantamientos tradicionales, como el press de banca, añade mayor estabilidad central y estabilización musculo-articular en relación con el peso corporal de la persona (Harrison, 2010).

Las progresiones en el entrenamiento de calistenia pueden ser un poco más desafiantes, pero la clave para una realización eficiente de los ejercicios con el peso corporal es la misma que con cualquier ejercicio, la técnica adecuada, el tiempo y la tensión (Harrison, 2010). Polo (2020) estima que, en calistenia, además de obtener ganancias de fuerza rápidamente, también vamos a adquirir progresos en cualidades como equilibrio, movilidad o flexibilidad, trabajando el cuerpo como un todo, de forma más funcional y global, lo que también la haga en el complemento ideal para otras modalidades deportivas. Por otra parte, en la práctica de la calistenia se dan ciertas desventajas, y es que, si no se tiene un mínimo de condición física, entrenar con nuestro propio peso corporal al comienzo no puede ser demasiado alentador.

Es cierto también, que si la pretensión es la hipertrofia (crecimiento de masa muscular), el entrenamiento con cargas externas parece ser más conveniente que la calistenia, lo cual no quiere decir que no se pueda construir masa muscular ni mucho menos, además, es difícil realizar un aislamiento de ciertos grupos musculares de manera más analítica, pues en este caso, las pesas o las máquinas nos ofrecen mayor variedad de ejercicios. Asimismo, el área más desfavorecida en la calistenia es el tren inferior. Así como en el gimnasio las progresiones se basan en incrementar la carga, es cierto que en calistenia dicha progresión es menos intuitiva y se ha de precisar de cierto conocimiento para "crear" combinaciones destinadas al objetivo incrementando la intensidad del entrenamiento.

Estas desventajas, no repercuten negativamente a este tipo de acondicionamiento, es más, cada una de las desventajas aludidas puede llegar a no ser entendida a lo largo de la práctica al observar la propia evolución, mejora y amplitud de conocimientos y ejercicios. Por su parte, Erdem y Genc (2020) reflejan en su artículo que la calistenia precisa de efectos positivos sobre una amplia variedad de parámetros en la composición corporal, VO2max, flexibilidad, potencia anaeróbica, capacidad aeróbica y rendimiento cardiovascular entre otros. Además, conciben 
que los ejercicios de calistenia favorecen a reducir la fatiga, mejorar el sueño, paliar problemas de concentración o mitigar la depresión, y, además, precisan de un papel elemental para hacer frente a patologías como la obesidad y la hiperglucemia.

Por consiguiente, vinculado con el entrenamiento de calistenia, a la par, también se destaca el entrenamiento interválico de alta intensidad (HIIT), pues teóricamente, la calistenia es una práctica de ejercicios rítmicos sin instrumentos concebidos como "ejercicios libres". De acuerdo con Alijas y Díaz (2015), en 1936 se pusieron en conocimiento las tres bases adoptadas para la estructuración de la calistenia, que fueron: selección, precisión y totalidad. Entre dichos principios, se concibió el principio de totalidad como pilar para desarrollar la tabla de referencia para las sesiones de calistenia con ejercicios divididos en tres grupos: introductorio (ejercicios de calentamiento), fundamental (ejercicios de extensión, flexión, flexión lateral de tronco, equilibrio, abdominales, carreras y saltos) y concluyente (ejercicios de vuelta a la calma).

De acuerdo con Fernandes et al. (2017), algunos investigadores han estado utilizando ejercicios de calistenia con entrenamiento en intervalos de alta intensidad (HIIT) y lo llaman calistenia HIIT, nueva calistenia o trabajo corporal HIIT (HBW). En consonancia con Scott et al. (2016), este tipo de entrenamiento se lleva a cabo a través de tiempo a alta intensidad, seguidos de cortos periodos de descanso, uno de los métodos más empleados dentro de este tipo de rutinas, es el Tabata, (Tabata, 2019) que comprende 8 series de 20 segundos de trabajo, teniendo 10 segundos de descanso entre cada una llevando a cabo ejercicios pliométricos.

Según Velasco-Orjuela et al. (2018), los efectos descritos tras efectuar una comparación entre el entrenamiento interválico con el entrenamiento extensivo aeróbico convencional, parece ser que este tipo de entrenamiento interválico tiene efectos similares e incluso mejores que el entrenamiento de resistencia extensivo en lo que respecta a cambios significativos óptimos en la composición corporal, reducción de porcentaje de grasa corporal, parámetros cardiorrespiratorios (mejora aptitud anaeróbica y aeróbica), factores fisiológicos (mayor tolerancia al lactato), aspectos cognitivos, disminución de concentraciones séricas de cortisol, disminución del colesterol y de las citocinas inflamatorias y mejora de control hormonal tanto anabólico como catabólico. Ahora bien, estos beneficios parecen ser reducidos en el tiempo o a pocas semanas y necesitan de adaptaciones constantes para ser efectivos (Guadalupe-Grau et al.,2017).

\section{CONCLUSIÓN}

La calistenia es un método de entrenamiento que lleva presente desde hace milenios y que ha sido empleada por nuestros ancestros como medio de entrenamiento para su preparación frente a un mundo que hoy en día ya no existe, pues sus habitantes disfrutan de un bienestar social determinado por la tecnología haciendo que el movimiento ya no sea necesario derivando a que las personas caigan en el sedentarismo y propiciando así diversas patologías relacionadas con la falta de movimiento que de antaño no acaecían.

La abundancia de recursos gracias al avance de la ciencia deportiva y el rol imperante que desempeñan las redes sociales en la actualidad, hacen que este tipo de método quede a un lado, siendo sustituido por otras formas de acondicionamiento físico más focalizadas hacia la práctica del culturismo por la predominancia de ciertos cánones estéticos tan arraigados a nivel 
social haciendo que cierto porcentaje poblacional acuda a centros fitness en los que se utiliza la imagen "perfecta" como reclamo.

Sin embargo, aunque el debate en el panorama actual entre si es mejor entrenar con cargas externas o corporales sea constante, este documento no tiene la pretensión de desacreditar o dar más valor a un tipo u otro de entrenamiento, sino de ofrecer una alternativa asequible para todo el mundo debido a la desigualdad existente en nuestra sociedad que lleva a que muchas personas no puedan permitirse pagar la mensualidad de un gimnasio. Asimismo, se trata de incentivar la crítica y la reflexión teniendo en cuenta el contexto por el que atraviesa la actividad física en la actualidad influenciado por los aspectos mencionados anteriormente.

La calistenia contiene tanto ventajas como desventajas, sin embargo, se necesita que sea más tratada en el ámbito de la investigación deportiva y de la Educación Física con el fin de establecer bases teóricas más consistentes para fomentar su práctica entre una población globalizada que parece haber olvidado su ancestral esencia. 


\section{REFERENCIAS}

Alijas, R. y Díaz, A. (2015). Calistenia: Volviendo a los orígenes. EmásF: Revista Digital de Educación Física, (33), 8796.

Coffey, V. G. \& Hawley, J. A. (2007). The molecular bases of training adaptation. Sports Med, vol (37), 737-763. DOI: 10.2165/00007256-200737090-00001

Cuadrado, G., Pablos. C. y García, J. (2006). Aspectos metodológicos y fisiológicos del trabajo de hipertrofia muscular. Wanceulen SL.

Erdem, A. \& Genc, H. (2020). The Effect of Calisthenics Exercises on Body Composition in Soccer Players. PROGRESS IN NUTRITION, vol (22), 94-102. DOI: 10.23751/pn.v22i1-S.9797

Fernandes A., Moreira, R.A., Gomes de Souza, R., Figueira, A. \& Sales, A. (2017). High intensity interval training with bodyweight: the new calisthenics? Manual Therapy, Posturology \& Rehabilitation Journal, vol (15), 1-4. DOI: 10.17784/mtprehabjournal.2017.15.448

González-Badillo, J. J., Marques, C. \& Sánchez-Medina, L. (2011). The importance of movement velocity as a measure to control resistance training intensity. J Hum Kinet, vol (29) 15-19. DOI: 10.2478 / v10078-011-0053-6

González-Badillo, J.J. y Gorostiaga. (2002). Fundamentos del entrenamiento de la fuerza. Inde

González-Badillo, J.J. y Ribas, J. (2002). Bases de la programación del entrenamiento de fuerza. Inde

Guadalupe-Grau, A., Fernández-Elías, V. E., Ortega, J. F., Dela, F., Helge, J. W., \& Mora-Rodriguez, R. (2017). Effects of 6-month aerobic interval training on skeletal muscle metabolism in middle-aged metabolic syndrome patients. Scandinavian Journal of Medicine \& Science in Sports, 28(2), 585-595. doi:10.1111/sms.12881

Harrison, J. (2010). Bodyweight Training: A Return To Basics, vol (32), 52-55.

Kraemer, W. J. \& Ratamess, N. A. (2004). Fundamentals of resistance training: progression and exercise prescription. Med Sci Sports Exerc, (vol) 36, 674-688. DOI: 10.1249/01.MSS.0000121945.36635.61

Polo, S. (2020). Valoración del orden óptimo entre una dominada completa y una straddel para un máximo rendimiento en calistenia. Trabajo de fin de grado. Universidad de Zaragoza. https://zaguan.unizar.es/record/98941

Sánchez, M. (2017). Factores determinantes del rendimiento en el ejercicio de dominadas y efectos del entrenamiento concurrente de fuerza y resistencia. Tesis doctoral. Universidad de Sevilla. Recuperado de: https://rio.upo.es/xmlui/handle/10433/4783?show=full

Scott, C., Beliveau, C., Desmond, K. \& Rollins, E. (2016). Total energy costs of 3 all-out Tabata routines: calisthenic, plyometric and resistance exercises. European Journal of Human Movement, vol (37), 49-55.

Tabata, I. (2019). Tabata training: one of the most energetically effective high-intensity intermittent training methods. The Journal of Physiological Sciences. doi:10.1007/s12576-019-00676-7

Tillin, N.A., Pain T.G. \& Folland, J. P. (2012) Contraction type influences the human ability to use the available torque capacity of skeletal muscle during explosive efforts. Proc. R. Soc. B, vol (279), 2106-2115 DOI: 10.1098/rspb.2011.2109

Velasco-Orjuela, G., Domínguez-Sánchez, M., Hernández, E., Correa-Bautista, J., Triana-Reina, H., García-Hermoso, A., Peña-Ibagón, J., Izquierdo, M., Cadore, A., Hackney, A. \& Ramírez-Vélez, R. (2018). Acute effects of highintensity interval, resistance or combined exercise protocols on testosterone - cortisol responses in inactive overweight individuals. Physiology \& Behavior, vol (194), 401-409. DOI: 10.1016/j.physbeh.2018.06.034 